\title{
Dispersion of Polarization in Reflection Nebulae
}

\author{
ThOMAS GeHRELS \\ University of Arizona \\ Tucson, Arizona
}

\begin{abstract}
A FEW OBSERVATIONS WERE MADE IN NGC 7023 with filters at 0.36 , $A_{0.56}$, and $0.74 \mu$. The wavelength dependence of polarization fitted with a straight line shows an appreciable rise with longer wavelengths. (See refs. 1 and 2.) The observations at the three filters were compared with Mie calculations obtained from Dr. B. M. Herman and General S. R. Browning of the University of Arizona for various refractive indices and particle sizes. Only single particle sizes have been considered, and therefore the results are preliminary. The present work represents a first reconnaissance to learn the techniques and some of the geometrical conditions.
\end{abstract}

A firm conclusion, however, is reached in ruling out purely graphitic and purely metallic interstellar grains. The reasoning is as follows: The polarizations in various reflection nebulae (observed by Elvius and Hall) are "positive" and strong, and the color dependence is strong, with a fairly steep rise toward longer wavelengths; this behavior occurs for scattering angles from $20^{\circ}$ to $70^{\circ}$. Such behavior is found in the Mie calculations at $2 \pi a / \lambda \cong 1.5$. (See ref. 3.) Diagrams similar to those in reference 3 were made in the present investigation for a large imaginary component added to the refractive index, and the conclusion is unchanged. The behavior is observed at $\lambda \cong 0.56 \mu$, so that $2 a \cong 0.3 \mu$ where $a$ is the particle radius. Such particle sizes fit the interstellar reddening and polarization-wavelength results exclusively for dielectric substances, and not for substances with a high imaginary component added to the refractive index, such as graphite and metallic particles (the latter would fit only for much smaller diameters, $2 a \cong 0.06 \mu$; see table III of ref. 4). This conclusion pertains to grains in the interstellar medium in general. Graphite nuclei might be generated near late-type stars and thus cause the peculiar polarizations of, for example, $\mu$ Cephei.

The Mie calculations were integrated over various ranges of the scattering angles, and self-extinction within the nebula was included. The cloud 
was assumed to be a uniform spherical nebula, and the particles were assumed to be spherical. Some of the photometry by Mme. T. M. Martel of France was also used. It is obviously important to combine polarimetry and photometry instead of treating them separately in these problems in which solutions must be made for many parameters.

Good fits of the theory and the observations have been obtained for both homogeneous particles with a refractive index of about 1.4, an imaginary component (between $0.1 i$ and $0.4 i$ ), and particle diameters of about $0.3 \mu$; and composite particles that have the outer shell with a refractive index of about 1.4, a negligible imaginary component, and a nucleus of diameter of about $0.06 \mu$ consisting of a metallic or graphitic substance.

If the star is in front of the reflection nebula, no fit can be obtained. The computed wavelength dependence is too steep regardless of the refractive index used. The conclusion drawn, therefore, is that the illuminating star (HD 200775) is either inside or behind the nebula. An estimate of about 0.5 is made of the visual albedo, and the directivity $<\cos \theta>$ is about 0.6 .

The distance to HD 200775 is 0.29 kiloparsec, and the space density in the nebula is about $4 \times 10^{-10}$ grains $/ \mathrm{cm}^{3}$.

In general, from the work on these problems and from the fits of the interstellar polarization observations, one gains the impression that the best fit is obtained for the coated grains: a condensation nucleus of a material with a high imaginary component included in the refractive index and having a diameter of the order of $0.06 \mu$, coated with a material of refractive index of about 1.4 with an imaginary component that is very small. A graphite core, coated with essentially pure ice to an outer diameter of about $0.3 \mu$, satisfies the requirements.

\section{REFERENCES}

1. Gehrels, Thomas; and Teska, Thomas M.: The Wavelength Dependence of Polarization. Appl. Opt., vol. 2, 1963, p. 67.

2. Gerrels, T.: Measurements of the Wavelength Dependence of Polarization. Lowell Obs. Bull., vol. 4, 1960, p. 300.

3. Van de Hulst, H. C.: Light Scattering by Small Particles. John Wiley \& Sons, Inc., 1957, pp. 152-153.

4. Gehrels, T.: The Wavelength Dependence of Polarization II Interstellar Polarization. Astron. J., vol. 65, 1960, p. 472.

\section{DISCUSSION}

Behr: Did you find any dependence of your observed curve on the apparent distance of the nebula point from the illuminating star?

Gehrels: No, we made calculations for only one distance from the central star. 
Behr: I do not understand how you draw these conclusions without knowing anything about the geometry of the nebula.

Gehrels: For the density estimate, the distance and the geometry must be known. However, for the polarimetry, there are two important factors involved. Let a scattering element having a scattering angle $\theta$ be the space confined by the observed beam, and consider discrete intervals of the scattering angle. With increasing $\theta$ the volume $V$ of the element decreases and the distance $R$ to the central star decreases. The weighting factor for the intensities $V / R^{2}$ is constant. This situation facilitates the use of the Mie calculations, and the value of $R$ does not have to be known.

The other factor that is important for the polarization work is the strong forward scattering of these particles. Only the range near small values of $\theta$ is important.

Wickramasinghe: I think I am right in assuming that the geometry doesn't enter. It is only a solid-angle effect.

Greenberg: Dr. Hall mentioned that there were approximately 1.5 magnitudes of extinction. Now, was any account taken of the extinguishing of the light that gets from the star to the particles and from the particles to the observer? This would play an important role. Furthermore, if the extinction is 1.5 magnitudes from the star in this spherical geometry, I would expect that there would be a considerable difference from the single scattering that you have done.

Wickramasinghe: I don't think this would affect the polarization, which is produced essentially by the cloud particles.

Greenberg: I will get to the polarization later, but that would certainly affect the amount of light, and I think it also would affect the amount of polarization because the contributions due to different parts would be differently affected-different angles have different polarization contributions. This particular $V / R^{2}$, which is simply a solid-angle effect, is fine, but there is an additional attenuation of the radiation coming from the different parts. Therefore, different angles of scattering have contributions which are modified by the different amounts of attenuation.

Gehrels: The extinction of the light from the star to the nebular particles has been accounted for. The nebula is small and the optical depth is small; therefore, only single scattering occurs.

The dimension of the nebula must be known only approximately, for some limitation on the range of scattering angles. It makes little difference whether we stopped at a scattering angle of $20^{\circ}$ or $10^{\circ}$.

The 1.5-magnitude extinction occurs mostly between the nebula and the observer, and it is the same for the starlight and for the particlescattered light. It does not affect the polarization, and the effect on brightness and color has been accounted for. 
Greenberg: Errors could also result from the interpretation in terms of single sizes. For example, in determining the wavelength dependence of polarization produced by infinitely alined cylinders, one finds for the typical Oort-van de Hulst size distribution that the value of $2 a$ is about 0.6. However, the single size of cylinder that would produce essentially the same type of polarization is a value of $2 a$ of about 0.4 . There is a difference factor of 2 in treating a single particle and a size distribution. This situation arises because the contribution at the various wavelengths is a different function of the size for polarization than it is for extinction.

Wickramasinghe: I would like to comment on the point which Professor Greenberg raised about the effect of grains outside the cloud. If you assume that the number density of spherical grains in the line of sight outside the cloud is much less than the number density inside the cloud, then Dr. Gehrels' conclusions follow.

Greenberg: In other words, the total extinction is inside the cloud.

Wickramasinghe: What really enters is the angle with the line of sight that the starlight reaches a grain. Inside the cloud all the grains are going to be scattering into different cones producing the polarization. The grains far away from the cloud in the line of sight receive the light at zero angle and, therefore, there is no interstellar polarization.

Greenberg: Yes, but the major part of the polarization is produced by the grains in the cloud. This is where I think he may be making a mistake in his calculations.

Voice: No, he is taking this into account. He is saying that all the stuff in the cloud is producing the polarization; isn't it?

Greenberg: Yes, it is on this basis that I say that the extinction within the cloud is ignored.

Voice: I also do not understand how you can draw conclusions regarding particle size. Why couldn't you decrease the calculated polarization from the 100 percent that you would get with isotropic Rayleigh scattering? Also, introducing a somewhat larger optical depth would tend to reduce the polarization.

Gehrels: The optical depth inside the cloud is very low and the multiple scattering is negligible.

Voice: Can it possibly fit with 1.5 magnitudes?

Gehrels: The 1.5-magnitude extinction occurs on the way; that is, from the observed cloud to the observer. The starlight and the nebular scattered light are affected by the same processes.

Elvius: Have you studied the thesis written by C. J. van Houten in 1961 at the Leiden University? Do you think that the optical depth of two magnitudes in the blue quoted by him is wrong?

Gehrels: Yes. The optical depth from nebula to the observer is large, but within the cloud the optical depth is small. 
Nandy: Why did you use the value 4.2 instead of 3.0 for the ratio of interstellar extinction to color excess?

Gehrels: This value was determined by $H$. L. Johnson.

Nandy: Do all your calculations depend upon this value?

Gehrels: The 4.2 value had nothing to do with the polarization conclusions.

Nandy: What about the distance of the star?

Gehrels: This factor did not affect the polarization conclusions, but did affect the density conclusions.

Nandy: Would the weighting factor $V / R^{2}=0.23$ be different if the distance were known?

Gehrels: Only in the first approximation, and it is of no consequence for the polarization work.

Elvius: As I pointed out, we found a lower polarization than you found. If your values were two-thirds lower, would that influence your conclusions?

Gehrels: It would affect the particle size; instead of the mean 0.30 , the grain diameter may be $0.24 \mu$. But this is well within the uncertainties of the measurements and of the analysis. After all, we are dealing with spherical particles. If the interstellar particles were snowflakes, for example, none of this would hold.

Wickramasinghe: How did you get these estimates of the optical depths?

Gehrels: The optical depth is obtained as the product of the scattering efficiency, the path length, the cross section, and the number density of the grains. A mean density through the nebula was adopted; and the nebula is assumed to be a uniform spherical one.

Donn: Your analysis suggests that polarization does not depend very much on the angle; this implies that polarization would not vary across the nebula.

Gehrels: I did not say that polarization is independent of the scattering angle; near $\theta=5^{\circ}$ or $\theta=20^{\circ}$ it does not make much difference whether the nebula is twice as large or not. We studied rather extreme scattering angles of $10^{\circ}$ and $20^{\circ}$, and the value of the angle did not affect the fits. However, for larger values of $\theta$, near $60^{\circ}$, the polarization depends strongly on $\theta$.

Donn: Do you have similar measurements at different distances from the central star at which you could carry out the same analysis?

Gehrels: No, but Elvius and Hall do. The distance is very important. For large values of $\theta$ near $90^{\circ}$ there is a very small range of scattering angles.

Nandy: How bright is the nebula?

Gehrels: I was working at about the 14th magnitude level with a diaphragm of $23^{\prime \prime}$ in diameter. The sky brightness was about one-third of the brightness of the spot. 